\title{
A UTILIZAÇÃO DO SISTEMA DE INDICADORES ASSISTENCIAIS POR GESTORES DE ENFERMAGEM DE UM HOSPITAL UNIVERSITÁRIO
}

\author{
Nathalia Barione Zancheta ${ }^{1}$, Rosana Rodrigues Figueira Fogliano ${ }^{2}$, Elena Bohomol${ }^{3}$, Vanessa Ribeiro Neves ${ }^{4}$, \\ Lúcia Marta Giunta da Silva ${ }^{4}$
}

\begin{abstract}
RESUMO: Os objetivos foram conhecer a utilização de sistema informatizado de indicadores de enfermagem de um hospital universitário do município de São Paulo, identificar a aplicabilidade dos resultados dos indicadores e verificar o retorno das informações para a equipe de enfermagem. Estudo descritivo, tipo survey, com abordagem quantitativa e população de 27 enfermeiros integrantes da Diretoria de Enfermagem. A coleta de dados ocorreu de maio a junho de 2014, por meio de um instrumento para avaliar a opinião desses gestores quanto à utilização do sistema. Evidenciou-se que $25(92,59 \%)$ dos sujeitos concordam que o sistema é de fácil manuseio, 13 (48,14\%) notificam eventos adversos e oito (29,63\%) não notificam. $12(44,44 \%)$ dos gestores utilizam o sistema para acompanhar a assistência prestada aos pacientes, entretanto, cinco (18,51\%) não apresentam os indicadores em reuniões periódicas para sua equipe. Os gestores utilizam os indicadores para planejar ações de melhoria, porém há fragilidades nas notificações de eventos adversos, demonstrando a necessidade de desenvolver a cultura da gestão de indicadores na instituição.
\end{abstract}

DESCRITORES: Indicadores de qualidade em assistência à saúde; Qualidade da assistência à saúde; Administração de serviços de saúde; Gestão da qualidade; Serviços de enfermagem.

\section{THE USE OF THE CARE INDICATORS SYSTEM BY NURSE MANAGERS AT A TEACHING HOSPITAL}

\begin{abstract}
The objectives were to get to know the use of a computerized nursing indicator system at a teaching hospital in the city of São Paulo, to identify the applicability of the indicator outcomes and to verify the information return for the nursing team. Descriptive survey with a quantitative approach and a population of 27 nurses, members of the Nursing Board. The data were collected between May and June 2014 through a survey of these managers' opinion on the use of the system. It was evidenced that $25(92.59 \%)$ subjects agree that the system is easy to handle. Thirteen $(48.14 \%)$ report adverse events while eight (29.63\%) do not. Twelve $(44.44 \%)$ managers use the system to monitor patient care, but five $(18.51 \%)$ do not present the indicators during periodical team meetings. The managers use the indicators to plan improvements, although frailties exist in the reporting of adverse events, demonstrating the need to develop the indicator management culture at the institution.
\end{abstract}

DESCRIPTORS: Quality indicators, health care; Quality of health care; Health services administration; Quality management; Nursing services.

\section{LA UTILIZACIÓN DEL SISTEMA DE INDICADORES ASISTENCIALES POR GESTORES DE ENFERMERÍA DE UN HOSPITAL UNIVERSITARIO}

RESUMEN: Los objetivos fueron conocer la utilización de sistema informatizado de indicadores de enfermería de un hospital universitario del municipio de São Paulo, identificar a aplicabilidad de los resultados de los indicadores y verificar el retorno de las informaciones para el equipo de enfermería. Estudio descriptivo, tipo survey, con aproximación cuantitativa y población de 27 enfermeros directores de Enfermería. Los datos fueron recolectados de mayo a junio del 2014 mediante un instrumento para evaluar la opinión de esos gestores respecto a la utilización del sistema. Fue evidenciado que $25(92,59 \%)$ de los sujetos concuerdan que el sistema es de fácil manoseo, 13 (48,14\%) notifican eventos adversos ocho (29,63\%) no notifican. 12 (44,44\%) gestores utilizan el sistema para acompañar la atención prestada a los pacientes, pero cinco (18,51\%) no presentan los indicadores durante reuniones periódicas para su equipo. Los gestores utilizan los indicadores para planificar acciones de mejora. Sin embargo, hay fragilidades en las notificaciones de eventos adversos, demostrando la necesidad de desarrollar la cultura de gestión de indicadores en la institución. DESCRIPTORES: Indicadores de calidad de la atención de salud; Calidad de la atención de salud; Administración de los servicios de salud; Gestión de la calidad; Servicios de enfermería.

${ }^{1}$ Enfermeira. Residente de Enfermagem na Saúde do Adulto e do Idoso da Escola de Enfermagem da Universidade de São Paulo. São Paulo, SP, Brasil.

${ }^{2}$ Enfermeira. Mestre em Enfermagem. Enfermeira da Escola Paulista de Enfermagem da Universidade Federal de São Paulo. São Paulo, SP, Brasil

${ }^{3}$ Enfermeira. Livre Docente. Docente da Escola Paulista de Enfermagem da Universidade Federal de São Paulo. São Paulo, SP, Brasil.

${ }^{4}$ Enfermeira. Doutora em Ciências. Docente da Escola Paulista de Enfermagem da Universidade Federal de São Paulo. São Paulo, SP, Brasil.

Autor Correspondente:

Rosana Rodrigues Figueira Fogliano

Universidade Federal de São Paulo

R. Napoleão de Barros, 754 - 04024-002 - São Paulo, SP, Brasil

E-mail: rosana.fogliano@unifesp.br
Recebido: 01/03/2016

Finalizado: 18/09/2016 


\section{INTRODUÇÃO}

A busca pela qualidade é um desafio constante para a manutenção da efetividade dos serviços de saúde e requer a utilização de ferramentas que permitam a identificação e monitorização da estrutura, dos processos e dos resultados das organizações e fundamentem ações de melhoria ao longo do tempo. Os dados numéricos que oferecem este panorama são os indicadores de qualidade ${ }^{(1)}$.

É crescente a preocupação dos enfermeiros com a construção, validação e utilização de indicadores que reflitam seu contexto de trabalho e subsidiem a melhoria da qualidade do serviço de Enfermagem nos cenários de assistência e de gestão de pessoas ${ }^{(2)}$.

Sistemas informatizados de indicadores de Enfermagem podem contribuir sobremaneira para a coleta, o armazenamento e a disponibilização, em tempo real, desses dados para análise, permitindo a avaliação dos resultados obtidos ${ }^{(3)}$ e, consequentemente, o gerenciamento dos resultados da assistência, favorecendo a melhoria contínua e a prestação dos cuidados com base em evidências ${ }^{(4)}$.

A correta utilização desses sistemas pelos membros da equipe de enfermagem torna-se crucial para a fidedignidade e o aproveitamento das informações obtidas ${ }^{(4)}$, particularmente por aqueles que ocupam cargos de chefia - como gerentes, coordenadores e encarregados. Deles emanam as diretrizes para utilização e a supervisão dos profissionais usuários do sistema, as ações educativas de capacitação permanente e, principalmente, o manejo dos dados obtidos em prol da realização de melhorias.

Diversos estudos descrevem a criação e implantação desses sistemas, desde a década de 1990 até os dias atuais ${ }^{(3,5)}$, mas são escassas as publicações sobre sua utilização pelos integrantes da equipe de enfermagem.

Diante desse cenário, questiona-se: de que maneira gerentes e encarregados de Enfermagem utilizam o sistema informatizado de indicadores? A resposta pode propiciar reflexão sobre o aproveitamento das informações oriundas desse sistema como subsídio para a tomada de decisão em Enfermagem, a prática baseada em evidências e os processos de melhoria contínua dos serviços de saúde.

Deste modo, os objetivos foram conhecer a utilização do sistema informatizado de indicadores de enfermagem pelos gerentes de serviços e encarregados de setores da Diretoria de Enfermagem de um hospital universitário do município de São Paulo, identificar a aplicabilidade dos resultados dos indicadores nas ações de melhoria da assistência prestada no hospital e verificar como ocorre o retorno das informações oriundas do sistema de indicadores para a equipe de enfermagem.

\section{- MÉTODO}

Estudo descritivo, do tipo survey, com abordagem quantitativa. Esse tipo de estudo permite coletar informações detalhadas de variáveis existentes e utilizar os dados para justificar e avaliar as condições de determinadas práticas, bem como propor planos de correção(6).

O estudo foi realizado num hospital universitário do município de São Paulo, terciário, de grande porte e alta complexidade, que possui 712 leitos. Atende prioritariamente pacientes do Sistema Único de Saúde (SUS) e tem por finalidade a assistência, o ensino e a pesquisa.

A população foi constituída pelos 27 enfermeiros ocupantes dos cargos de gerentes de enfermagem e encarregados de setores integrantes da Diretoria de Enfermagem do hospital, representando a totalidade dos profissionais que ocupam tais cargos na instituição e que utilizam o sistema informatizado de indicadores.

Determinou-se como critério de inclusão enfermeiros gerentes e encarregados das unidades que utilizavam o Sistema Informatizado de Indicadores, e como critério de exclusão gerentes e encarregados de unidades com tempo inferior a três meses no cargo.

A coleta de dados ocorreu em maio e junho de 2014, por meio da aplicação de um instrumento contendo questões de caracterização dos participantes e concernentes à utilização do sistema de indicadores do hospital. Para responder sobre utilização dos indicadores, foi utilizada uma escala 
tipo Likert, com cinco graus de variação [Concordo totalmente; Concordo; Nem concordo, nem discordo; Discordo; e Discordo totalmente]. Esse instrumento foi elaborado pelas pesquisadoras e avaliado por três enfermeiros com experiência em Gestão de Serviços de Enfermagem e na utilização de indicadores, sendo dois docentes de Administração em Enfermagem e um gerente de instituição assistencial. As mudanças propostas por esses avaliadores foram discutidas e incorporadas à versão final do instrumento, que foi entregue aos sujeitos e respondida por todos.

Os dados coletados foram armazenados e organizados numa planilha do programa Microsoft Excel ${ }^{\circledR}$, submetidos à análise estatística descritiva, apresentados em frequência relativa e absoluta e discutidos à luz da literatura disponível sobre o tema.

Todos os sujeitos foram convidados a participar voluntariamente da pesquisa e assinaram o Termo de Consentimento Livre e Esclarecido. A realização do estudo foi aprovada em 23/4/2014 pelo Comitê de Ética em Pesquisa da Universidade Federal de São Paulo, conforme parecer $n^{\circ}$ 63.026, e pela Diretoria de Enfermagem do hospital.

\section{RESULTADOS}

Dos 27 gerentes e encarregados, todos atenderam aos critérios de inclusão. Destes, sete (25,93\%) ocupavam o cargo de gerentes de serviços e 20 (74,07\%) de encarregados de setores da Diretoria de Enfermagem do hospital estudado. A mediana de idade dos encarregados foi de 36 e dos gerentes 38 anos. Houve predominância do sexo feminino, 23 (85,19\%), o tempo médio de formado foi de 15 anos para os gerentes e 11 anos para os enfermeiros encarregados e 18 (66,67\%) sujeitos ocupam o cargo atual há pelo menos oito anos.

Grande parte dos enfermeiros, $23(85,19 \%)$, recebeu capacitação prévia para utilizar o Sistema Informatizado de Indicadores e 19 (70,37\%) relataram fazê-lo diariamente. Na Tabela 1, observa-se que $25(92,59 \%)$ consideraram o sistema de fácil manuseio; somente $13(48,15 \%)$ informaram que suas equipes notificam eventos adversos e oito $(29,63 \%)$ que ainda não notificam.

Tabela 1 - Opinião dos sujeitos sobre o sistema de indicadores ( $\mathrm{n}=27)$. São Paulo, SP, Brasil, 2016

\begin{tabular}{|c|c|c|c|c|c|c|c|c|c|c|}
\hline \multirow[t]{2}{*}{ Afirmativas } & \multicolumn{2}{|c|}{$\begin{array}{l}\text { Concordo } \\
\text { totalmente }\end{array}$} & \multicolumn{2}{|c|}{ Concordo } & \multicolumn{2}{|c|}{$\begin{array}{c}\text { Nem } \\
\text { concordo, } \\
\text { nem discordo }\end{array}$} & \multicolumn{2}{|c|}{ Discordo } & \multicolumn{2}{|c|}{$\begin{array}{l}\text { Discordo } \\
\text { totalmente }\end{array}$} \\
\hline & $n$ & $\%$ & $\mathrm{n}$ & $\%$ & $n$ & $\%$ & $\mathbf{n}$ & $\%$ & $\mathbf{n}$ & $\%$ \\
\hline $\begin{array}{l}\text { Considero o sistema de indicadores da } \\
\text { assistência de enfermagem utilizado no } \\
\text { hospital de fácil manuseio. }\end{array}$ & 13 & 48,15 & 12 & 44,44 & 2 & 7,41 & 0 & 0 & 0 & 0 \\
\hline $\begin{array}{l}\text { A minha equipe notifica eventos adversos } \\
\text { relacionados aos Indicadores de Qualidade } \\
\text { nas unidades. }\end{array}$ & 3 & 11,11 & 10 & 37,04 & 6 & 22,22 & 8 & 29,63 & 0 & 0 \\
\hline $\begin{array}{l}\text { Avalio as notificações de eventos adversos } \\
\text { ocorridos nas unidades diariamente. }\end{array}$ & 4 & 14,81 & 10 & 37,04 & 5 & 18,52 & 7 & 25,93 & 1 & 3,7 \\
\hline $\begin{array}{l}\text { Utilizo o sistema de indicadores para } \\
\text { acompanhar a assistência prestada aos } \\
\text { pacientes. }\end{array}$ & 3 & 11,11 & 9 & 33,33 & 11 & 40,74 & 4 & 14,81 & 0 & 0 \\
\hline $\begin{array}{l}\text { Analiso mensalmente os dados gerados } \\
\text { pelos indicadores. }\end{array}$ & 7 & 25,93 & 13 & 48,15 & 4 & 14,81 & 2 & 7,41 & 1 & 3,7 \\
\hline $\begin{array}{l}\text { Apresento para minha equipe as informações } \\
\text { obtidas através dos indicadores após análise } \\
\text { em reuniões periódicas. }\end{array}$ & 3 & 11,11 & 8 & 29,63 & 11 & 40,74 & 4 & 14,81 & 1 & 3,7 \\
\hline $\begin{array}{l}\text { Utilizo os resultados obtidos por meio dos } \\
\text { indicadores para planejar ações de melhoria. }\end{array}$ & 2 & 7,41 & 16 & 59,26 & 7 & 25,93 & 2 & 7,41 & 0 & 0 \\
\hline $\begin{array}{l}\text { Realizo análise comparativa dos dados dos } \\
\text { indicadores depois das ações de melhoria. }\end{array}$ & 1 & 3,7 & 9 & 33,33 & 13 & 48,15 & 4 & 14,81 & 0 & 0 \\
\hline
\end{tabular}


Adicionalmente, 14 (51,85\%) afirmaram avaliar essas notificações, enquanto oito $(29,63 \%)$ não o fazem; apenas $12(44,44 \%)$ relataram utilizar o sistema para acompanhar a assistência prestada aos pacientes e quatro $(14,81 \%)$ afirmaram não utilizá-lo. Destaca-se que 11 (40,74\%) não manifestaram sua concordância ou discordância a respeito da utilização do sistema e, contrapondo-se a esse resultado, $20(74,07 \%)$ afirmaram analisar mensalmente os dados obtidos por meio do sistema.

A maioria dos sujeitos, 16 (59,25\%), não apresentou os resultados em reuniões periódicas para suas equipes, entretanto $18(66,67 \%)$ afirmaram utilizar essas informações para planejar ações de melhoria. E, ainda, 17 (62,96\%) não realizaram análise comparativa dos indicadores após ações de melhoria.

\section{DISCUSSÃO}

Os participantes têm perfil semelhante ao encontrado na literatura que aponta a enfermagem como uma profissão predominantemente feminina ${ }^{(7-8)}$ e com correlação positiva entre o tempo de formação e atuação no serviço e a aquisição de experiência para exercer cargos de liderança ${ }^{(7,9)}$. Os resultados evidenciaram que a função gerencial é exercida por profissionais formados há mais de 10 anos e que atuavam há pelo menos oito anos na instituição, em consonância com o Regimento Interno de Enfermagem do hospital, cujos critérios para ocupação dos cargos de gerente e encarregado é ter no mínimo cinco anos e dois anos de atuação na instituição respectivamente.

Sabe-se que a formação do enfermeiro possui lacunas quanto à identificação e aplicação dos indicadores na prática profissional, de modo que o treinamento para utilizar o sistema informatizado de indicadores, tanto do ponto de vista técnico quanto conceitual, torna-se imprescindível para a concretização da cultura de qualidade, que inclui a notificação de eventos adversos ao paciente ${ }^{(10)}$.

Considerando que a maioria dos gestores recebeu treinamento para uso do sistema e o consideraram de fácil manuseio, chama atenção que a utilização dos resultados obtidos não alcance percentuais tão altos. Destaca-se que, embora os sujeitos refiram analisar os dados e avaliar as notificações, a utilização dessas informações como sinalizadores da necessidade de ações de melhoria ainda não está consolidada em todos os líderes. Este fato corrobora com estudos que demonstram as dificuldades dos enfermeiros em transformarem sua prática profissional mediante a análise e comparação de resultados obtidos por meio de indicadores de qualidade ${ }^{(11-12)}$.

Esse cenário indica a fragilidade dos processos que envolvem a segurança do paciente e poderiam ser aprimorados pelo aproveitamento das informações disponíveis no sistema. A constatação de que os gestores não apresentam os resultados para suas equipes e não realizam análises comparativas, para acompanhar o andamento da qualidade do serviço de enfermagem, demonstra a lacuna existente entre as evidências oriundas da prática e a tomada de decisão desses profissionais.

\section{CONSIDERAÇÕES FINAIS}

O presente estudo permitiu conhecer a utilização do sistema informatizado de indicadores de enfermagem pelos 27 enfermeiros gerentes e encarregados da Diretoria de Enfermagem de um hospital universitário do município de São Paulo.

Apesar das dificuldades encontradas, os gestores utilizaram os resultados obtidos por meio dos indicadores para planejar ações de melhoria. Isto reforça a necessidade de intervenções educacionais, uma vez que não se observa a consolidação do conhecimento, em toda a equipe de enfermeiros, sobre gerenciamento da qualidade com foco na segurança do paciente.

Podemos observar que a cultura da gestão dos indicadores na instituição ainda não está bem sedimentada, necessitando de estratégias futuras de implementação com foco na qualidade e segurança do paciente e esses resultados poderão subsidiar a elaboração de ações de melhoria na gestão da instituição.

Concluiu-se que as notificações de eventos adversos ainda apresentam fragilidade no desempenho de atividades pelos enfermeiros gestores, que devem focar nas ações junto à equipe sob sua 
responsabilidade, a fim de aumentar essas notificações e o compartilhamento das informações obtidas, após análise dos indicadores, em reuniões periódicas para a equipe de enfermeiros.

Cabe ressaltar que este estudo retrata a realidade de um hospital universitário da cidade de São Paulo, com um Sistema Informatizado de Indicadores próprio. Sugere-se, portanto, sua replicação em outros contextos.

\section{REFERÊNCIAS}

1. Agência Nacional de Vigilância Sanitária (ANVISA). Assistência Segura: uma reflexão teórica aplicada à prática. Série: Segurança do Paciente e Qualidade em Serviços de Saúde. Brasília: ANVISA; 2013.

2. Programa de Qualidade Hospitalar (CQH). Manual de Indicadores de Enfermagem NAGEH. [Internet] São Paulo: APM/CREMESP; 2012 [acesso em 19 mai 2016]. Disponível:

http://www.cqh.org.br/portal/pag/doc.php?p_ndoc=125.

3. Labbadia LL, D'Innocenzo M, Fogliano RRF, Silva GEF, de Queiroz RMRM, Carmagnani MIS, et al. Sistema informatizado para gerenciamento de indicadores da assistência de Enfermagem do Hospital São Paulo. Rev. esc. enferm. USP. 2011; 45(4): 1013-7.

4. Vituri DW, Évora YDM. Gestão da Qualidade Total e enfermagem hospitalar: uma revisão integrativa de literatura. Rev. bras. enferm. 2015; 68(5): 945-52.

5. Kagan I, Cohen R, Fish M, Mezare HP. Developing and implementing a computerized nursing quality control system in a tertiary general medical center in Israel. J. nurs. care qual. 2014; 29(1): 83-90.

6. Gil AC. Métodos e técnicas de pesquisa social. São Paulo: Atlas; 1999.

7. Santos I, Castro CB. Características pessoais e profissionais de enfermeiros com funções administrativas atuantes em um Hospital Universitário. Rev. esc. enferm. USP. 2010; 44(1): 154-60.

8. Trigueiro EV, Leite JEL, Dantas DNA, Coura AS, Enders BC. Perfil e posicionamento do enfermeiro gerente quanto ao processo de enfermagem. Esc. Anna Nery. 2014; 18(2): 343-9.

9. Nóbrega MFB, Matos MG, da Silva LMS, Jorge MSB. Perfil gerencial de enfermeiros que atuam em um hospital público federal de ensino. Rev. enferm. UERJ. 2008; 16(3): 333-8.

10. de Menezes PIFB, D'Innocenzo M. Dificuldades vivenciadas pelo enfermeiro na utilização de indicadores de processos. Rev. bras. enferm. 2013; 66(4): 571-7.

11. Silveira TVL, Prado Júnior PP, Siman AG, Amaro MOF. Opinião dos enfermeiros sobre a utilização dos indicadores de qualidade na assistência de enfermagem. Rev. gaúcha enferm. 2015; 36(2): 82-8.

12. Rossaneis MA, Gabriel CS, Haddad MCFL, Melo MRAC, Bernardes A. Indicadores de qualidade utilizados nos serviços de enfermagem de hospitais de ensino. Rev. eletr. enf. [Internet] 2014; 16(4): 769-76 [acesso em 20 jan 2016]. Disponível: http://dx.doi.org/10.5216/ree.v16i4.22956. 